\title{
Linc HOTAIR epigenetically silences miR34a by binding to PRC2 to promote the epithelial-to-mesenchymal transition in human gastric cancer
}

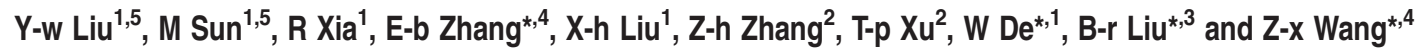

IncRNAs play important roles in the epigenetic regulation of carcinogenesis and progression. Previous studies suggest that HOTAIR contributes to gastric cancer (GC) development, and the overexpression of HOTAIR predicts a poor prognosis. In this study, we found that HOTAIR was more highly expressed in diffuse-type GC than in intestinal type $(P=0.048)$. In the diffuse type, there is significant relationship between HOTAIR expression and DFS $(P<0.001)$. CDH1 was downregulated in diffuse-type GC tissues $(P=0.0007)$ and showed a negative relationship with HOTAIR $\left(r^{2}=0.154, P=0.0354\right)$. In addition, HOTAIR knockdown significantly repressed migration, invasion and metastasis both in vitro and vivo and reversed the epithelial-to-mesenchymal transition in GC cells. We also showed that HOTAIR recruiting and binding to PRC2 epigenetically represses miR34a, which controls the targets C-Met (HGF/C-Met/Snail pathway) and Snail, thus contributing to GC cell-EMT process and accelerating tumor metastasis. Moreover, it is demonstrated that HOTAIR crosstalk with microRNAs during epigenetic regulation. Our results suggest that HOTAIR acts as an EMT regulator and may be a candidate prognostic biomarker and a target for new therapies in GC patients. Cell Death and Disease (2015) 6, e1802; doi:10.1038/cddis.2015.150; published online 2 July 2015

Gastric cancer is the fourth most frequent cancer and the second leading cause of cancer-related deaths worldwide. ${ }^{1}$ The poor prognosis of patients with gastric cancer is largely due to the high frequency of tumor recurrence or metastasis after surgical resection. ${ }^{2}$ Chemotherapy and molecularly targeted therapy are the main treatments for advanced gastric cancer. Therefore, a better understanding of the early events associated with gastric cancer metastasis is warranted to decrease mortality and improves patient's quality of life.

In the past decades, cell and tumor biologists have identified the key role of epithelial-mesenchymal transition (EMT) in cancer cell invasion and metastasis, a biological process where epithelial cells lose their polarity and undergo transition into a mesenchymal phenotype. ${ }^{3}$ Recent evidence revealed that EMT could enhance cancer cell invasion by promoting Rac-dependent mesenchymal migration, and also contributes to cancer cell proliferation and survival. ${ }^{4,5}$ Generally, the important hallmarks of EMT include the loss of E-cadherin and increased expression of non-epithelial cadherins, such as vimentin and $\mathrm{N}$-cadherin. The loss of $\mathrm{E}$-cadherin expression is a fundamental event in EMT process and a crucial step in the progression of papillomas to invasive carcinomas. ${ }^{6}$ There are studies demonstrated that epigenetic changes, such as microRNAs (miRNAs), histone modifications and DNA methylation, are involved in cancer cell EMT. ${ }^{7-9}$ For example, miR34a inhibits the epithelial-to-mesenchymal transition and cancer cell migration. ${ }^{10}$ In the colorectal cancer miR34a enhances Snail expression and activates IL-6 R/STAT3 signaling to induce EMT. ${ }^{11,12}$ Meanwhile, our previous study found that long non-coding RNA (IncRNA) BANCR contributes to non-small lung cancer cell invasion and metastasis via regulating EMT. ${ }^{13}$

It is estimated that $98 \%$ of the human genome transcripts are non-coding RNAs (ncRNAs), which form a highly complex

\footnotetext{
${ }^{1}$ Department of Biochemistry and Molecular Biology, Nanjing Medical University, Nanjing, Jiangsu, PR China; ${ }^{2}$ Department of Pathology, First Affiliated Hospital of Nanjing Medical University, Nanjing, Jiangsu, PR China; ${ }^{3}$ Department of Oncology, Affiliated Drum Tower Hospital of Nanjing University, Nanjing, Jiangsu, PR China and ${ }^{4}$ Department of Oncology, Second Affiliated Hospital of Nanjing Medical University, Nanjing, Jiangsu, PR China

*Corresponding author: W De, Department of Biochemistry and Molecular Biology, Nanjing Medical University, Road hanzhong 140, Nanjing, Jiangsu 210029, PR China. Tel/Fax: +86 258686 2728; E-mail: dewei@njmu.edu.cn

or B-r Liu, Department of Oncology, Affiliated Drum Tower Hospital of Nanjing University, Nanjing, Jiangsu 210008, PR China. Tel/Fax: +86 2583304616 ; E-mail: nibrliu@163.com

or Z-x Wang, Department of Oncology, Second Affiliated Hospital of Nanjing Medical University, Nanjing, Jiangsu 210011, PR China. Tel/Fax: +86 25 58509900 ; E-mail: zhaoxiawang88@ hotmail.com

${ }^{5}$ These authors contributed equally to this work.

Abbreviations: LncRNA, long non-coding RNA; HOTAIR, HOX antisense intergenic RNA; miR34a, microRNA34a; PRC2, polycomb repressive complex 2; EMT, epithelial-to-mesenchymal transition; PcG, polycomb group protein; BANCR, BRAF-activated non-coding RNA; ncRNA, non-coding RNA; EZH2, enhancer of zest homolog 2; H3K27me3, histone H3 lysine-27 trimethylation; HGF, hepatocyte growth factor; qRT-PCR, quantitative real-time polymerase chain reaction; DFS, disease-free survival; HE, hematoxylin and eosin; GSEA, gene set enrichment analysis; GEO, Gene Expression Omnibus; MALAT-1, metastasis-associated lung adenocarcinoma transcript 1; ZEB1, zinc-finger E-box-binding homeobox 1; ZEB2, zinc-finger E-box-binding homeobox 2; WHO, The World Health Organization; UICC, Union for International Cancer Control; GAPDH, glyceraldehyde-3-phosphate dehydrogenase; IHC, immunohistochemistry; SDS-PAGE, sodium dodecyl sulfate-polyacrylamide gel electrophoresis; PVDF, polyvinylidene fluoride; PMSF, phenylmethanesulfonyl fluoride; TBS, Tris-buffered saline; ChIP, chromatin immunoprecipitation; RIP, RNA immunoprecipitation; PBS, phosphate-buffered saline; DMEM, Dulbecco's modified Eagle's medium; CT, cycle threshold; BSA, bovine serum albumin; ECM, extracellular matrix; SiRNA, small interfering RNA; ShRNA, short hairpin RNA; GC, gastric cancer; IL6R, interleukin 6 receptor; STAT3, signal transducer and activator of transcription 3; TWIST, Transaction Workflow Innovation Standards Team; CDK, cyclin-dependent kinase

Received 13.2.15; revised 30.3.15; accepted 08.4.15; Edited by E Candi
} 
regulatory network and have diverse biological functions in tumor genesis. ${ }^{14}$ IncRNAs are important new members of the ncRNA family that are greater than $200 \mathrm{nt}$ without protein coding ability. Recently, researchers have linked the aberrant IncRNA expression with diverse human diseases, in particular cancers. ${ }^{15,16}$ Therefore, identification of gastric cancerassociated IncRNAs and investigation of their molecular mechanisms in controlling EMT are important in understanding the molecular biology of gastric cancer metastasis and progression. The IncRNA HOX antisense intergenic RNA (HOTAIR) was first identified in $2007 .{ }^{17}$ Recently, lots of studies have shown that HOTAIR is overexpressed in colorectal cancer, pancreatic cancer, breast cancer and gastrointestinal stromal tumors and is positively correlated with a poor clinical outcome. ${ }^{18-21}$ The activity of HOTAIR is partially due to its interaction with the polycomb repressive complex 2 (PRC2; EZH2, SUZ12 and EED), which enhances histone H3 lysine-27 trimethylation of the HOXD locus to decrease multiple gene expression from HOXD. ${ }^{17}$ Our previous study showed that HOTAIR expression is increased in gastric cancer tissues and is associated with malignant characteristics and poor prognosis. Furthermore, HOTAIR promotes gastric cancer cell proliferation in vivo and in vitro by competing 'sponge' miR-331-3p. ${ }^{22}$ However, the molecular mechanisms of HOTAIR involved in gastric cancer cell metastasis remain largely unknown.

In this study, we found that HOTAIR is more highly expressed in diffuse-type gastric cancer than in intestinaltype gastric cancer and is negatively related to E-cadherin. High expression of HOTAIR in diffuse-type gastric cancer predicted poor DFS. Additional experiments revealed that HOTAIR knockdown significantly repressed migration, invasion and metastasis both in vitro and in vivo and reversed the gastric cancer cell EMT. In addition, HOTAIR also epigenetically downregulates miR34a by binding to PRC2 to activate its target genes C-Met (HGF/C-Met/Snail pathway) and Snail, thereby promoting EMT in advanced stages of gastric cancer. Our findings provide new insights into the mechanisms by which IncRNAs regulate the expression of miRNAs.

\section{Results}

HOTAIR and CDH1 expression levels in human gastric cancer tissue. The diffuse type has stronger metastasis behavior than the intestinal-type gastric cancer. We previously determined that HOTAIR expression was significantly upexpression in cancer tissues. ${ }^{22}$ In this study, the human gastric cancer tissues were histopathologically classified into intestinal $(n=35)$ and diffuse types $(n=26)$. HOTAIR expression was significantly higher in the diffuse-type gastric cancer $(P=0.048)$ compared to the intestinal-type gastric cancer (Figure 1a). Examination of the correlation between HOTAIR expression and clinical pathological features showed that HOTAIR upregulation was correlated with lymph node metastasis and vasculature invasion (Table 1). For diseasefree survival, patients with high HOTAIR expression had a significantly poorer prognosis than those with low HOTAIR expression for the diffuse-type gastric $(P<0.001$, log-rank test; Figure 1b); however, there was no significant correlation between HOTAIR expression and outcome for the intestinal- type gastric cancer (Figure 1c). $C D H 1$ is a vital metastasis marker in gastric cancer. We detected $\mathrm{CDH} 1$ expression by qPCR and immunohistochemistry. $C D H 1$ expression was downexpression in cancerous tissues $(P<0.001)$ compared to the normal tissue, and was negative for $60 \%$ of the diffusetype gastric cancer tissues (Figures $1 \mathrm{~d}$ and e). In addition, HOTAIR was negatively correlated with $C D H 1\left(r^{2}=0.154\right.$, $P=0.0354$; Figure 1f). These results indicate that HOTAIR overexpression play an important role in metastasis and may be useful for the development of novel prognostic or progression markers for advanced gastric cancer.

Modulation of HOTAIR expression in gastric cancer cells. To investigate the effect of HOTAIR on the invasion and metastasis of gastric cancer cells, we first examined the expression levels of HOTAIR, miR34a and EMT markers in various cancer cell lines by qRT-PCR. As shown in Supplementary Figure S1A, of the five gastric cancer cell lines (SGC-7901, BGC-823, MGC-803, AGS and MNK45), BGC-823 expressed higher levels of HOTAIR (4.11-fold) and lower levels of miR34a (0.11-fold) and $C D H 1(0.17$-fold) than the normal gastric epithelium cell line (GES-1); however, SGC-7901 expressed relative lower HOTAIR and higher miR34a and $C D H 1$ expression. Therefore, we chose SGC-7901 and BGC-823 as the experimental cell lines. The results showed that HOTAIR expression was effectively knocked down in BGC-823 and SGC-7901 cells by siHOTAIR1\# and si-HOTAIR2\# (Figure 2a), which were subsequently used in the further experiments. The efficiency of the sh-HOTAIR was shown in Supplementary Figure S2A.

HOTAIR promotes gastric cancer cell invasion and metastasis in vitro and in vivo. To investigate the effect of HOTAIR on the gastric cancer cell migration and invasion, Transwell assays were performed and the results revealed that inhibition of HOTAIR decreased BGC-823 and SGC-7901 cell invasion and migration (Figure 2b). To investigate the metastasis-promoting function of HOTAIR in vivo, we used a tail vein assay. Transwell assays certificated that the sh-HOTAIR1\# and sh-HOTAIR2\# treatment both decreased the invasion and migration of BGC-823 cells (Supplementary Figure S2B). Western blot assays certificated that the sh-HOTAIR1\# treatment increased the epithelial marker E-cadherin in BGC-823 cells (Supplementary Figure S2C). Furthermore, sh-HOTAIR stably transfected BGC-823 cells were injected mice tail vein. Seven weeks after injection, the mice were killed and the lung tissues were collected. As expected, the sh-HOTAIR group exhibited a lower frequency of lung metastases and displayed less weight loss compared to the pENTR vector group (Figure 2c). This difference was further confirmed following examination of the entire lungs by hematoxylin and eosin (HE) staining of lung sections (Figure 2d). Taken together, these results indicate that HOTAIR possesses metastasis-promoting activity and that its upregulation may facilitate the metastasis of gastric cancer cells.

HOTAIR promotes metastasis of gastric cancer cells by inducing EMT. Next, we investigated how HOTAIR facilitates metastasis of gastric cancer cells. As EMT is a critical 

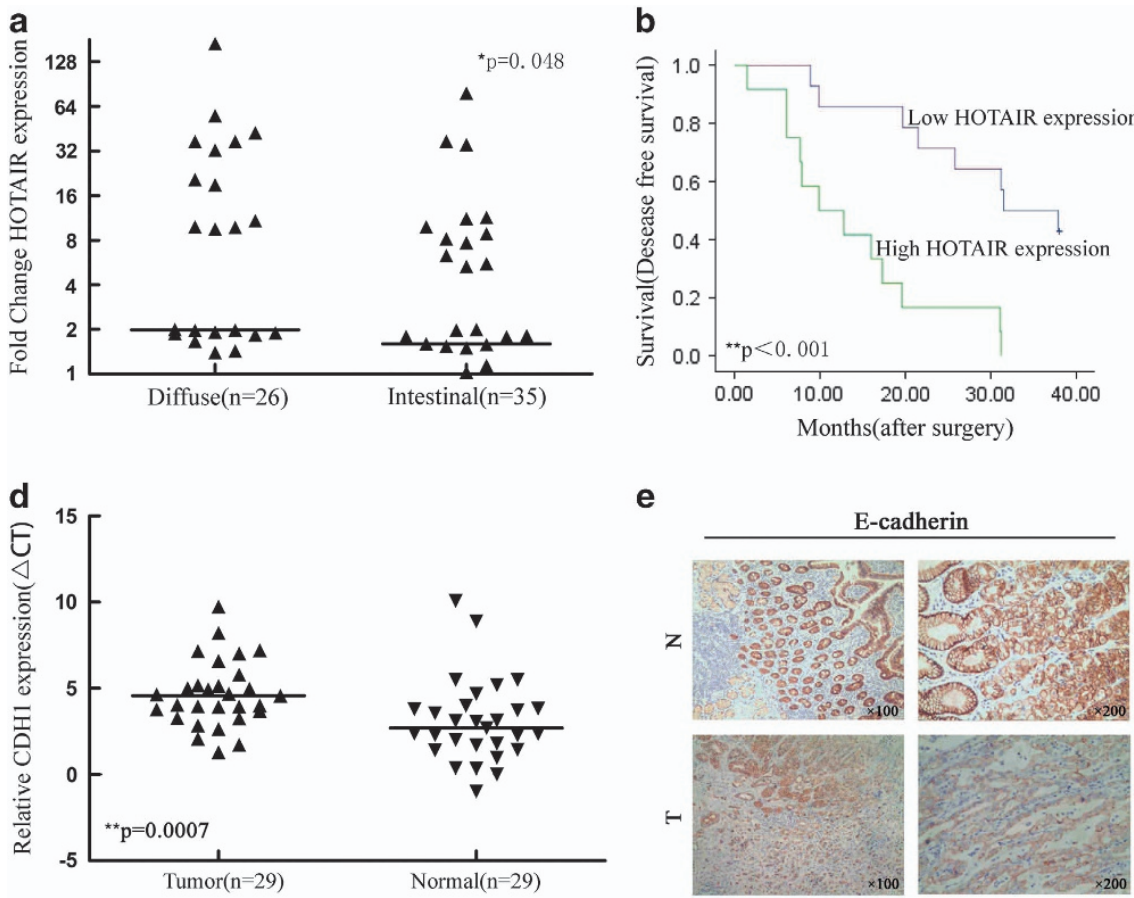

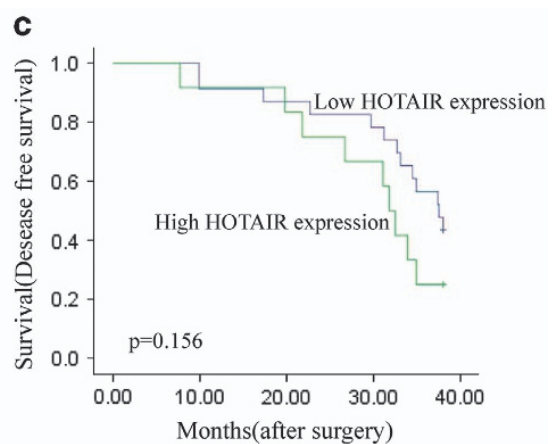

f

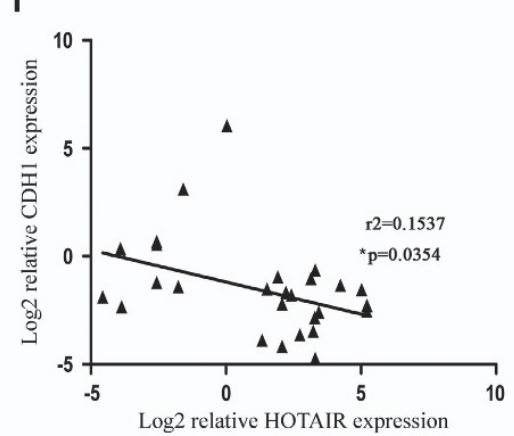

Figure 1 Relative HOTAIR expression in gastric cancer tissues and its clinical significance. (a) Relative expression of HOTAIR in the intestinal type of gastric cancer ( $n=35$ ) compared to diffuse-type gastric cancer $(n=26)$. HOTAIR expression was evaluated by qRT-PCR and normalized to GAPDH expression. Final results were presented as fold change in tumor tissues relative to normal tissues. Fold change is $\geq 2.0$ for high expression and $<2.0$ for low expression. The data are presented as the fold change in the intestinal tissues relative to the diffuse tissues. (b and $\mathbf{c}$ ) Kaplan-Meier disease-free survival curves according to the HOTAIR expression levels in different gastric cancer types. (d) Relative expression of CDH1 in diffuse-type gastric cancer compared to normal tissue $(n=29)$. (e) Upper: E-cadherin immunostaining in normal tissue samples. Lower: E-cadherin immunostaining is negative in diffuse-type gastric cancer tissues. (f) Correlation analysis between the expression of HOTAIR and E-cadherin in 29 paired tumors. ${ }^{*} P<0.05$ and ${ }^{*} P<0.01$

Table 1 Correlation of the expression of HOTAIR with clinicopathologic features in gastric cancer

\begin{tabular}{|c|c|c|c|c|}
\hline \multirow[t]{2}{*}{ Characteristics } & \multirow[t]{2}{*}{$N(\%)$} & \multicolumn{2}{|c|}{ HOTAIR $^{a}$} & \multirow[t]{2}{*}{$P$-value } \\
\hline & & High & Low & \\
\hline $\begin{array}{l}\text { Gender } \\
\text { Male } \\
\text { Female }\end{array}$ & $\begin{array}{l}38(62) \\
23(38)\end{array}$ & $\begin{array}{r}17 \\
7\end{array}$ & $\begin{array}{l}21 \\
16\end{array}$ & 0.295 \\
\hline $\begin{array}{l}\text { Age } \\
\quad \leq 65 \\
\quad>65\end{array}$ & $\begin{array}{l}31(51) \\
30(49)\end{array}$ & $\begin{array}{l}11 \\
13\end{array}$ & $\begin{array}{l}20 \\
17\end{array}$ & 0.605 \\
\hline $\begin{array}{l}\text { Stage } \\
\text { I-II } \\
\text { III-IV }\end{array}$ & $\begin{array}{l}18(30) \\
43(70)\end{array}$ & $\begin{array}{r}4 \\
20\end{array}$ & $\begin{array}{l}14 \\
23\end{array}$ & 0.092 \\
\hline $\begin{array}{l}\text { Lymph node metastasis } \\
\quad \leq 2 \\
\quad>2\end{array}$ & $\begin{array}{l}22(36) \\
39(64)\end{array}$ & $\begin{array}{r}5 \\
19\end{array}$ & $\begin{array}{l}17 \\
20\end{array}$ & $0.05^{\star}$ \\
\hline $\begin{array}{l}\text { Vasculature invasion } \\
\text { Yes } \\
\text { No }\end{array}$ & $\begin{array}{l}28(46) \\
33(54)\end{array}$ & $\begin{array}{r}18 \\
6\end{array}$ & $\begin{array}{l}10 \\
27\end{array}$ & $0.05^{\star}$ \\
\hline
\end{tabular}

${ }^{\star} P<0.05$ was considered significant (chi-square test between the two groups). ${ }^{a}$ Fold change (FC; tumor tissues relative to normal tissues) is $\geq 2.0$ for high expression and $<2.0$ for low expression.

event that contributes to tumor metastasis, we evaluated the effect of HOTAIR on gastric cancer cell-EMT process. First, we observed that BGC-823 and SGC-7901 cells transfected with si-HOTAIR partly restored epithelial cell polarity showing cobblestone-like morphology (Supplementary Figure S1C). Then, we detected the mRNA and protein expression levels of molecular markers of EMT following HOTAIR knockdown. The expression of the epithelial marker E-cadherin $(C D H 1)$ was higher in HOTAIR knockdown cells compared to the control cells. By contrast, the mesenchymal markers, including $\mathrm{N}$-cadherin and vimentin, were decreased in the HOTAIR knockdown BGC-823 and SGC-7901 cells (Figures 2e and f). Furthermore, we obtained the same results used stably transfected cells that the expression of E-cadherin was increased in sh-HOTAIR cells compared to the control cells; however, the $\mathrm{N}$-cadherin and vimentin were decreased in shHOTAIR in BGC-823 cells (Supplementary Figure S2C). These results suggest that HOTAIR may promote gastric cancer cell metastasis by inducing EMT.

HOTAIR downregulates the miR34a in gastric cancer. Recently, mounting evidences have shown that IncRNAs potentially regulate other classes of ncRNAs, including miRNAs. To investigate whether HOTAIR could regulate miRNA expression, we used Gene Set Enrichment Analysis (GSEA) software (Massachusetts Institute of Technology, Cambridge, MA, USA) to analyze the GES47638 data and predict the potential underlying miRNA of HOTAIR downstream. We found that various miRNAs may be HOTAIR downstream, and Supplementary Figure S1D shows target genes of six miRNAs, which were significantly enriched downstream of HOTAIR. Next, to investigate whether these 

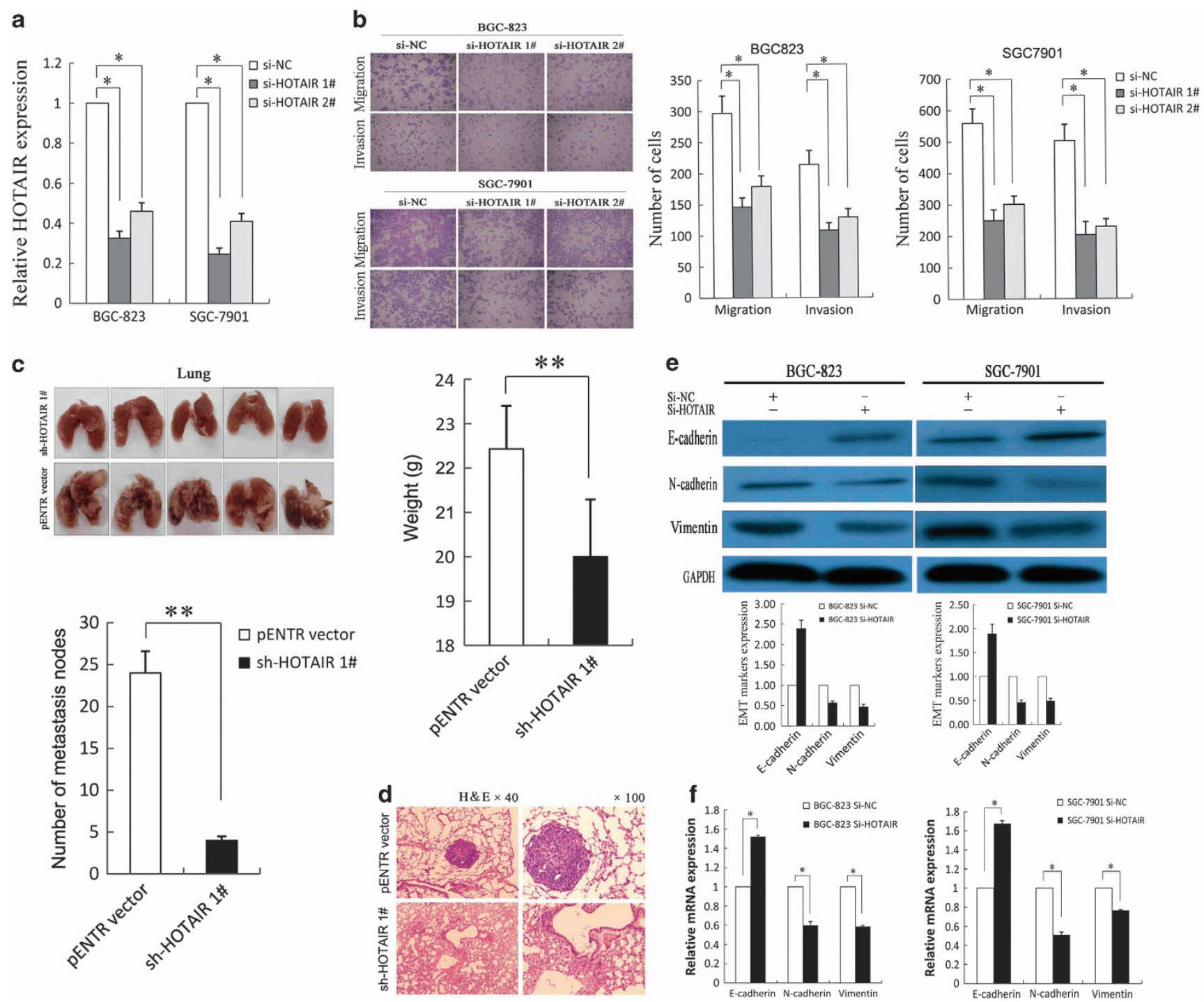

Figure 2 HOTAIR-promoted gastric cancer cell invasion, metastasis and EMT. (a) qRT-PCR was used to detect HOTAIR expression of BGC-823 and SGC-7901 cells with si-HOTAIRs. (b) Transwell assays were used to investigate the changes in migratory and invasive abilities of GC cells. (c) The lungs from mice in each experimental group with the numbers of tumor nodules on lung surfaces and weight were shown. (d) Visualization of the entire lung and HE-stained lung sections. (e and f) Western blotting and qRT-PCR were performed for analysis of E-cadherin, N-cadherin and vimentin in BGC-823 and SGC-7901 cells with si-HOTAIR. All experiments were performed in triplicate with three technical replicates. ${ }^{*} P<0.05$ and ${ }^{* *} P<0.01$

miRNAs could be regulated by HOTAIR in gastric cancer cells, we subsequently detected miRNAs in si-HOTAIRtransfected BGC-823 or SGC-7901 cells and found that miR34a expression was upregulated by 4.2 -fold compared with control cells $(P<0.01$, Figures $3 a$ and $b)$. Then we detected miR34a expression in gastric cancer tissues and found that miR34a expression was decreased in tumor tissue (Figure $3 \mathrm{c}$ ) and negatively correlated with HOTAIR expression in diffuse and intestinal types $\left(r^{2}=0.857\right.$ and 0.702 , $P<0.001$; Figures $3 \mathrm{~d}$ and $e$ ). These data indicated that HOTAIR may downregulate miR34a expression and promote gastric cancer cell metastasis.

HOTAIR silenced miR34a expression by recruiting PRC2. It is reported that $20 \%$ of the IncRNAs can bind the polycomb group protein (PcG) complex to regulate downstream gene transcription. EZH2, a key subunit of PRC2, which also includes SUZ12 and EED is a histone methyltransferase and represses downstream gene transcription by trimethylating histone H3 lysine 27 (H3K27me3). ${ }^{23,24}$ To determine whether HOTAIR regulates miR34a expression levels by binding with PRC2, we used ENCODE Histone Modification Tracks embedded in UCSC Genome Browser (UC Santa Cruz, CA, USA) and found H3K27me3 enrichment peaks in the miR34a promoter region (Figure 4a). Furthermore, we verified that HOTAIR was located both in the nucleus and cytoplasm of gastric cancer cells (Supplementary Figure S1B), and RNA immunoprecipitation (RIP) assays showed that HOTAIR could bind to PRC2 (Figure 4b). We knocked down EZH2 and SUZ12 by si-RNA in BGC-823, MGC-803 and SGC-7901 cells (Figures $4 \mathrm{c}$ and $\mathrm{d}$ ) and demonstrated that miR34a was upregulated compared to the controls; however, miR375 expression was not changed compared to control (Supplementary Figure S3A). The results of chromatin 
a

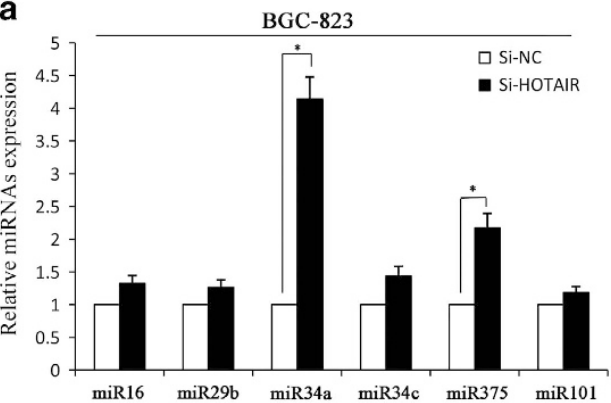

b

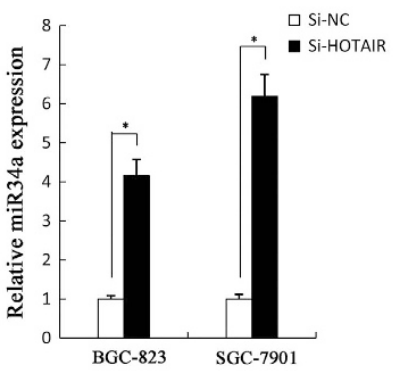

C

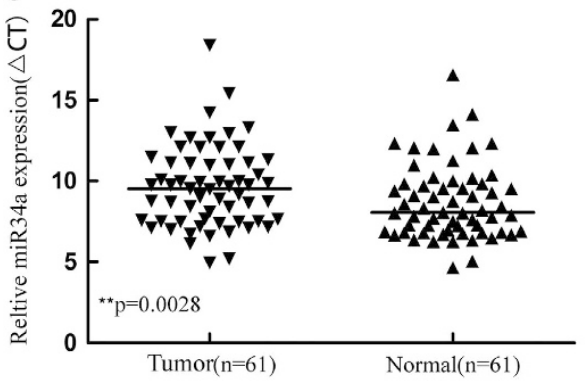

d

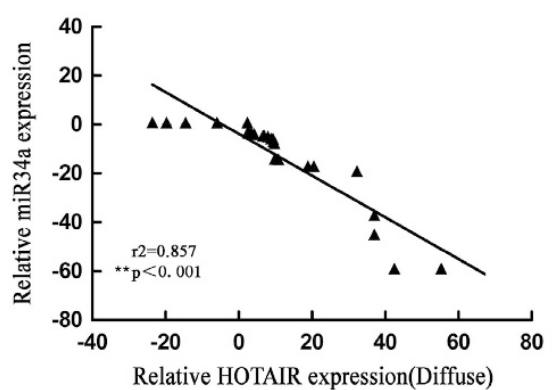

e

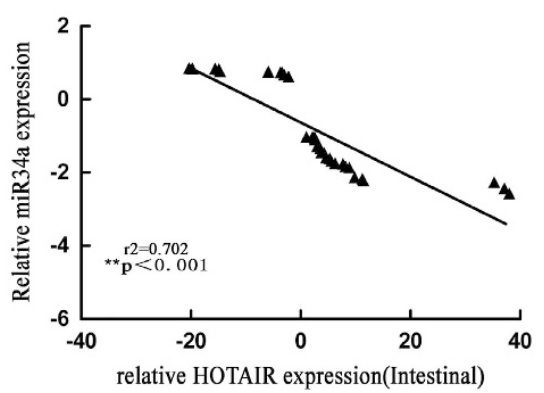

Figure 3 Correlation between the expression of HOTAIR and the miR34a. (a and $\mathbf{b})$ MiR34a expression was detected in BGC-823 and SGC-7901 cells with si-HOTAIR by qRT-PCR. All experiments were performed in triplicate with three technical replicates. (c) The expression level of miR34a in 61 paired tumors and peritumoral gastric cancers was detected by qRT-PCR. (d and e) Correlation analysis between the expression of HOTAIR and miR34a was examined in diffuse- and intestinal-type gastric cancer tissues. ${ }^{*} P<0.05$ and ${ }^{* *} P<0.01$

a

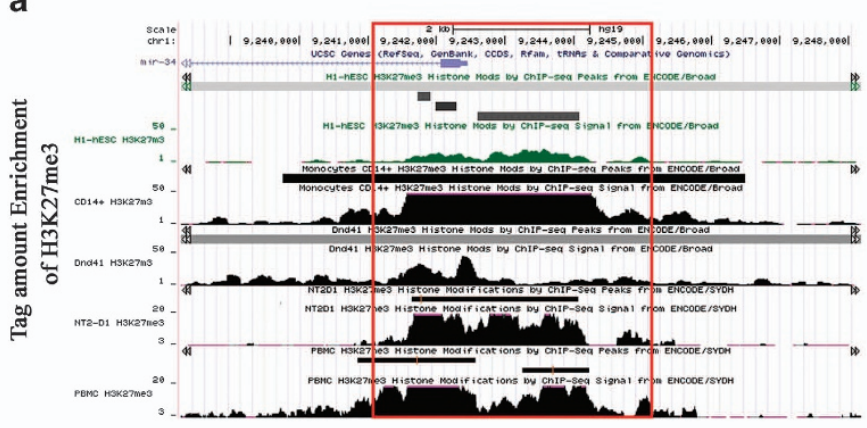

b

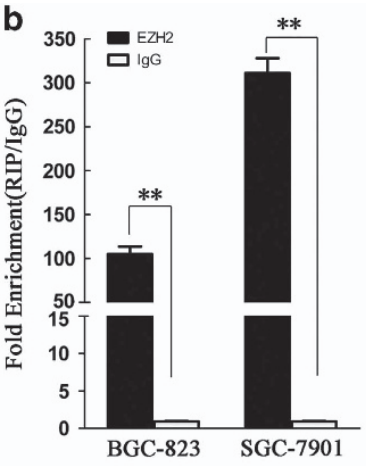

c

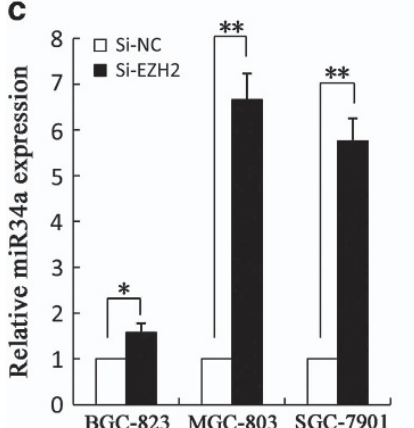

d

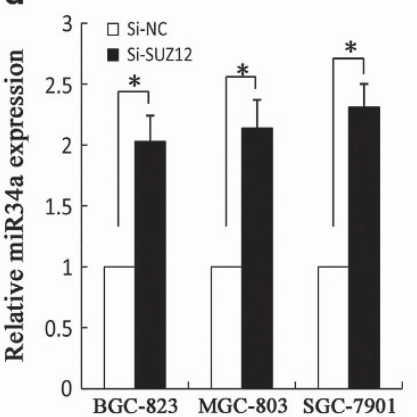

e

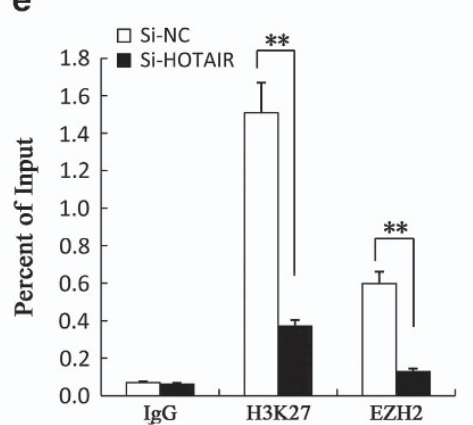

$\mathbf{f}$

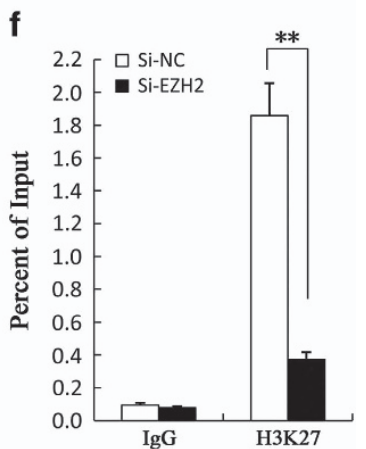

Figure 4 The association of HOTAIR with the PRC2 protein complex is critical for its regulation of miR34a. (a) Genome browser and analyzed H3K27me3 enrichment peaks in the miR34a promoter region. (b) RIP experiments were performed using the EZH2 antibodies for immunoprecipitation. Specific primers for HOTAIR were used to detect HOTAIR. (c and d) Expression of miR34a in BGC-823, SGC-7901 and MGC-803 cells transfected with si-EZH2, si-SUZ12 was detected by qRT-PCR. (e and f) ChIP analyses in SGC-7901 transfected with Si-HOTAIR and Si-EZH2 cells were performed on the miR34a promoter regions using anti-H3K27me3 and EZH2 antibodies. Enrichment was determined relative to the input controls. All experiments were performed in triplicate with three technical replicates. ${ }^{*} P<0.05$ and ${ }^{* \star} P<0.01$ 
immunoprecipitation (ChIP) assays showed that EZH2 could directly bind to the promoter region of miR34a and mediate H3K27me3 modification, while knockdown of HOTAIR and $E Z H 2$ led to reduced EZH2 and H3K27 binding ability (Figures $4 \mathrm{e}$ and f). In conclusion, these data indicate that HOTAIR recruit the PRC2 complex to silence miR34a via H3K27me3 modification.

\section{Upregulation of HOTAIR-enhanced gastric cancer cell} metastasis via activating HGF/Met/Snail pathway. Next, we investigated the role of miR34a in HOTAIR-induced EMT and metastasis promotion. MiR34a mimics were transfected into BGC-823 and SGC-7901 cells to upregulate miR34a expression, and the qPCR showed that miR34a expression was increased in miR34a mimics transfected gastric cancer cells (Figure 5a). Transwell assays revealed that the restoration of miR34a expression significantly inhibited the migration of BGC-823 and SGC-7901 cells (Figure 5b). HGF/Met/Snail pathway plays a critical role in EMT and metastasis, which was found to be regulated by $\operatorname{miR} 34 a^{25-28}$ Consistently, the restoration of miR34a in BGC-823 and SGC-7901 cells significantly reduced the protein levels of its targets C-Met and Snail, while upregulated E-cadherin and downregulated $\mathrm{N}$-cadherin and vimentin (Figure $5 \mathrm{c}$ ). Immunofluorescence analysis also revealed that enhanced miR34a expression decreased Snail expression not effected its subcellular localization in gastric cancer cells (Supplementary Figure S3C). By contrast, co-transfect of miR34a mimics and pcDNA-HOTAIR in BGC-823 and SGC-7901 cells partly reversed C-Met, Snail, E-cadherin, $\mathrm{N}$-cadherin and vimentin expression (Figures $5 \mathrm{~d}$ and $\mathrm{e}$ ). These data suggest that

HOTAIR is mechanistically linked to increased gastric cancer cell metastasis via dependent of miR34a. a

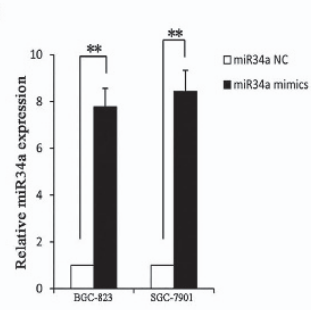

C

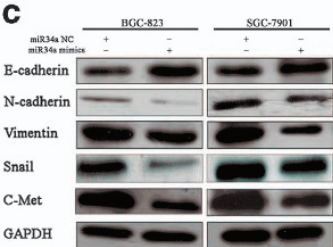

\section{b}
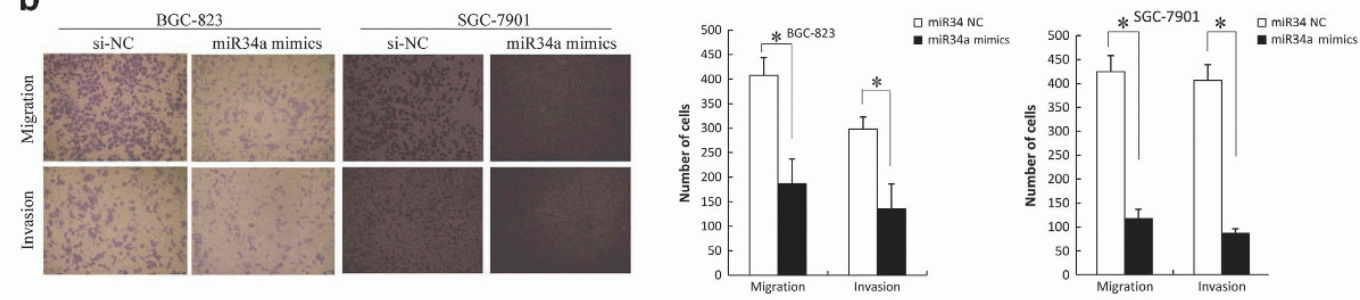

d
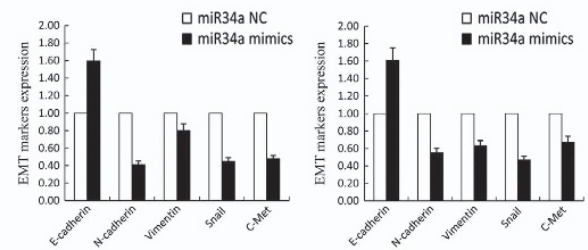
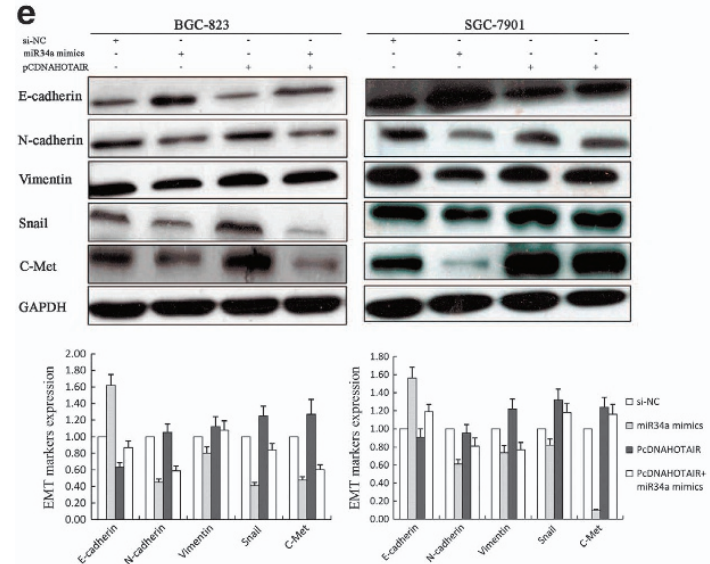

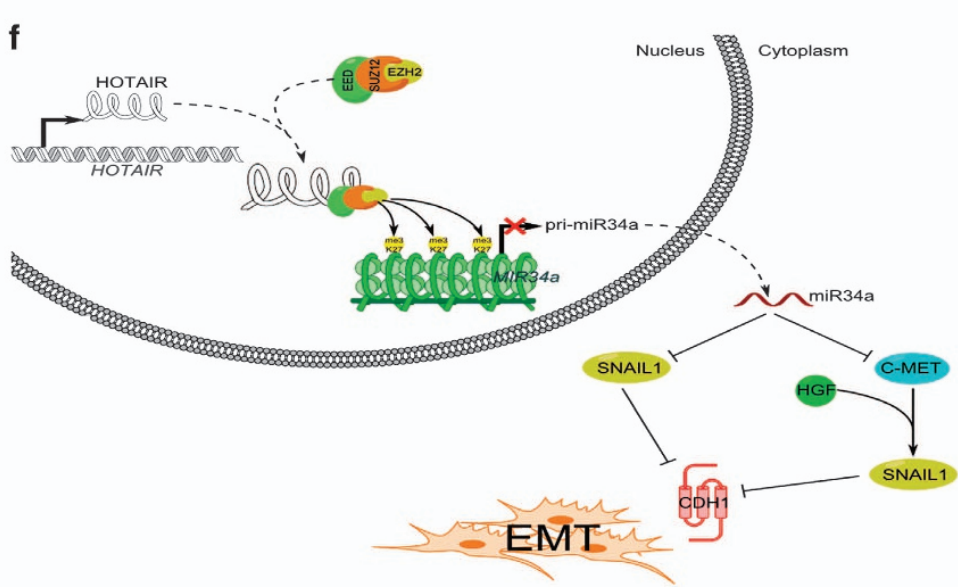

Figure 5 HOTAIR induces EMT by silencing miR34a in gastric cancer. (a) qRT-PCR was used to detect miR34a expression of BGC-823 and SGC-7901 cells with miR34a mimics. (b) Transwell assays were used to investigate the changes in the migratory and invasive abilities of gastric cancer cells with miR34a mimics. (c) Western blot assays were performed to detect the protein of C-Met, Snail and EMT markers in BGC-823 and SGC-7901 cells with miR34a mimics. GAPDH was used as a control. (d and e) Western blot assay and qRT-PCR were performed for analysis of E-cadherin, N-cadherin and vimentin; C-Met and Snail in BGC-823 and SGC-7901 cells. All experiments were performed in triplicate with three technical replicates. ${ }^{*} P<0.05$ and ${ }^{* *} P<0.01$. (f) Binding of HOTAIR to PRC2 leads to epigenetically silencing miR34a, downexpression of miR34a facilitated C-Met transcription, which active HGF/C-Met/ to induce Snail gene transcription; in addition, miR34a directly regulates Snail translation, which repressed E-cadherin transcription to promote EMT in gastric cancer 


\section{Discussion}

The incidence and mortality of gastric cancer have decreased significantly over the past 50 years worldwide; however, there remain large numbers of gastric cancer patients with poor prognosis for relapse and metastasis, particularly with diffusetype gastric cancer in Asia. ${ }^{29}$ Recently, a study showed that nearly $76 \%$ of the GENCODE (The National Human Genome Research Institute, Bethesda, MD, USA) annotated IncRNAs was differentially expressed between gastric cancer and normal gastric tissue. This suggests that IncRNAs may play vital roles in carcinogenesis and may be useful for discovery of new biomarkers and therapeutic targets in gastric cancer. ${ }^{30}$ For example, $\mathrm{H} 19$ is upregulated in gastric cancer and its overexpression is correlated with gastric cancer patient's poor prognosis, and enhances carcinogenesis and metastasis. ${ }^{31}$ In this study, our results revealed that high levels of HOTAIR expression were associated with venous invasion, diffusetype gastric cancer and poor DFS. These findings suggest that HOTAIR has a vital role in aggressive and metastatic and may be a novel metastatic or prognostic marker for gastric cancer.

Recent studies have shown that EMT is an important molecular mechanism involved in cancer cell metastasis and progression. Hallmarks of EMT are the loss of E-cadherin expression and upregulation expression of $\mathrm{N}$-cadherin and vimentin. $^{32-35}$ EMT is initiated by transcription factors or external signals, such as Snail, Twist, Zeb and HGF. ${ }^{36,37}$ In addition to these signaling pathways triggered by membrane receptors, recent studies have highlighted the importance of ncRNAs in the regulation of the epithelial phenotype by controlling EMT inducers. ${ }^{38}$ MiR200 is one of the major positive regulators in the maintenance of the epithelial phenotype via repression of ZEB1 ${ }^{39}$ Furthermore, the IncRNA MALAT-1 promoted the EMT by regulating ZEB1, ZEB2 and Slug expression and activating Wnt signaling. ${ }^{40}$ In this study, we demonstrated that downregulation of HOTAIR expression contributes to the significant inhibition of cell migration, invasion and metastasis. Moreover, decreased levels of HOTAIR expression resulted in significant increased expression of E-cadherin but decreased expression of $\mathrm{N}$-cadherin and vimentin in gastric cancer cells. These results demonstrate that HOTAIR mediates gastric cancer cell migration and metastasis, which also may be via affecting EMT.

Recently, mounting evidences have shown that IncRNAs potentially interact with other classes of ncRNAs, including miRNAs, and epigenetically regulate the expression of multiple genes, including those involved in chromatin modification, transcription and post-transcriptional processing. For example, HOTAIR functions as a 'CeRNA' to regulate HER2 expression by sponging $\mathrm{miR}-331-3 p$ to promote proliferation in gastric cancer. ${ }^{22,41,42}$ Approximately, 20\% of the IncRNAs can recruit the PcG complexes to regulate gene expression. ${ }^{43}$ $\mathrm{EZH} 2$ could epigenetically silence the downstream gene transcription, and it is overexpressed in several types of cancer, including gastric cancer. ${ }^{44-46}$ In this study, we demonstrated that HOTAIR recruits and binds to PRC2 to epigenetically silence miR34a expression to promote gastric cancer cell-EMT process and metastasis.

In addition, miR34a expression was downregulated and negatively correlated with HOTAIR expression in gastric cancer tissues. The identified targets of miR34a include CDK4/6, cyclin E2 (CCNE2), cyclin D1 (CCND1), E2F3, c-Met and Snail, ${ }^{47-49}$ and HGF/C-Met /Snail pathway plays an important role in EMT and cancer cell metastasis. ${ }^{27,38,50}$ HGF binding to its receptor c-Met leads to autophosphorylation and then activate the Ras/ Raf/MAPK pathway, which induces Snail gene transcription. ${ }^{27}$ Simultaneously, miR34a directly regulates Snail translation by binding to its $3^{\prime}$ UTR and inhibiting protein coding. ${ }^{11}$ In this study, restoration of miR34a significantly inhibited the migration and invasion capacity of gastric cancer cells in vitro, and suppressed the EMT of gastric cancer cells by targeting Met and Snail. In addition, upregulation of HOTAIR could partially reversed mi34a overexpression-mediated C-Met, E-cadherin, $\mathrm{N}$-cadherin, vimentin and snail expression.

LncRNAs have been proposed as potential targets for prognosis and therapeutic intervention. We describe here a novel mechanism by which HOTAIR reduced expression of miR34a: (i) HOTAIR recruited and binded to PRC2 epigenetically silencing miR34a; (ii) which in turn activate C-Met and as a consequence resulting in the activation of Snail transcription; and (iii) upregulated Snail expression promotes EMT in gastric cancer (Figure 5f). The prognostic significance of HOTAIR expression will help us to better predict the risk of recurrence. Finally, these data provide new insights into the RNA regulation network, indicating that IncRNAs not only target proteins but also affect miRNA expression through chromatin modification. Individual therapy targeting both miR34a and HOTAIR may lead to improved treatment.

\section{Materials and Methods}

Tissue samples. In this study, matched tumor tissues and adjacent non-tumor tissues were obtained from 61 gastric cancer patients at the Department of Surgical Oncology Jiangsu Province People's Hospital, Nanjing Medical University and Subei People's Hospital from March 2011 to December 2011. Two pathologists evaluated all specimens according to the World Health Organization (WHO) guidelines and the PTNM Union for International Cancer Control (UICC) pathological staging criteria. No local or systemic treatments were administered to these patients before surgery. The tissues were immediately frozen in liquid nitrogen and stored at $-80^{\circ} \mathrm{C}$ until use. Informed consent was obtained from all patients. The Human Research Ethics Committee of Jiangsu Province People's Hospital and Subei People's Hospital approved the study.

Total RNA extraction quantitative real-time polymerase chain reaction. Total RNA was extracted from the cultured cells and frozen tissues using TRIzol reagent (Invitrogen, Karlsruhe, Germany) following the manufacturer's protocol. Quantitative real-time polymerase chain reaction (PCR) was performed to detect HOTAIR and miR34a using the PrimeScript RT reagent kit and SYBR Premix Ex Taq (TaKaRa, Dalian, China) according to the manufacturer's instructions. The results were normalized to the expression of glyceraldehyde-3-phosphate dehydrogenase (GAPDH) or U6. The specific primers used are presented in additional file 3: Supplementary Table S1. The qPCR and data collection were performed on ABI 7500 (Applied Biosystems, Carlsbad, CA, USA). The qPCR results were analyzed and expressed relative to the $\mathrm{CT}$ (threshold cycle) values and then converted to fold changes; 2.0 -fold change was considered significant. ${ }^{18}$

Plasmid generation. The HOTAIR sequence was synthesized and subcloned into the pCDNA3.1 (Invitrogen, Shanghai, China) vector. Ectopic expression of HOTAIR was achieved via pCDNA-HOTAIR transfection, with an empty pCDNA3.1 vector used as a control. We also synthesized shRNA sequence-targeted HOTAIR. Si-HOTAIR sequence removed five bases of the $3^{\prime}$ end were converted to sh-HOTAIR. After annealing of the complementary shRNA oligonucleotides, we cloned the annealed oligonucleotides into pENTR vector (sh-HOTAIR; additional file 3 : Supplementary Table S1). The expression levels of HOTAIR were detected by qPCR. 
Immunohistochemistry. Paraffin-embedded, formalin-fixed human gastric tumor tissues were immunostained for the E-cadherin proteins. The signal was amplified and visualized using 3,30-diaminobenzidine chromogen followed by counterstaining with hematoxylin. Expression was considered positive when $10 \%$ or more of the cancer cells were stained. Anti-E-cadherin $(1: 50)$ was purchased from Cell Signaling Technology (CST, Danvers, MA, USA).

Cell culture. The BGC-823 and MGC-803 lines were cultured in RPMI 1640 medium (Gibco, CA, USA) containing $10 \%$ fetal bovine serum and incubated at $37^{\circ} \mathrm{C}, 5 \% \mathrm{CO}_{2}$ and saturated humidity. The SGC-7901 cells were cultured in DMEM medium (Gibco, CA, USA) containing 10\% fetal bovine serum and incubated at $37^{\circ} \mathrm{C}, 5 \% \mathrm{CO}_{2}$ and saturated humidity. Cell growth was observed under an inverted microscope. Cells in the logarithmic growth phase were harvested for the experiments.

Cell transfection. Plasmid vectors (pCDNA3.1-HOTAIR and pCDNA3.1) for transfection were prepared using DNA Midiprep or Midiprep kits (Qiagen, Hilden, Germany) and transfected into BGC-823 or SGC-7901 cells. The si-HOTAIR, sh-HOTIR, si-EZH2, si-SUZ12, miR34a mimics or si-NC were transfected into BGC-823 and SGC-7901 cells (additional file 3: Supplementary Table S1). BGC-823 or SGC-7901 cells were grown on six-well plates to confluency and transfected using Lipofectamine 2000 (Invitrogen) according to the manufacturer's instructions. At $48 \mathrm{~h}$ post transfection, cells were harvested for qPCR or western blot analysis.

Cell migration and invasion assays. For the migration assays, at $48 \mathrm{~h}$ post transfection, $5 \times 10^{4}$ cells in serum-free media were placed into the upper chamber of an insert (8- $\mu \mathrm{m}$ pore size; Millipore, Bedford, MA, USA). For the invasion assays, $1 \times 10^{5}$ cells in the serum-free medium were placed into the upper chamber of an insert coated with Matrigel (Sigma-Aldrich, St. Louis, MO, USA). Medium containing $10 \%$ FBS was added to the lower chamber. After incubation for $24 \mathrm{~h}$, the cells remaining on the upper membrane were removed with cotton wool. Cells that had migrated or invaded through the membrane were stained with methanol and $0.1 \%$ crystal violet, imaged and counted in five random fields per well using an IX71 inverted microscope (Olympus, Tokyo, Japan). Experiments were independently repeated three times.

Western blotting analysis and antibodies. Cell lysates were prepared using RIPA protein extraction reagent (Beyotime, Beijing, China) supplemented with a protease inhibitor cocktail (Roche, Basel, Switzerland) and phenylmethylsulfonyl fluoride (Roche). Approximately, $50 \mu \mathrm{g}$ of protein extract was separated by $10 \%$ sodium dodecyl sulfate-polyacrylamide gel electrophoresis (SDS-PAGE) and then transferred onto a polyvinylidene fluoride (PVDF) membrane (Millipore). GAPDH was used as a control. Antibodies (1:1000) against E-cadherin and $\mathrm{N}$-cadherin were purchased from BD (Franklin Lakes, NJ, USA). Antibodies $(1: 1000)$ against vimentin and C-MET were purchased from Cell Signaling Technology (CST). An antibody (1:1000) against Snail was purchased from Abcam (Cambridge, UK).

Subcellular fractionation location. The separation of nuclear and cytosolic fractions was performed using the PARIS kit (Life Technologies, Grand Island, NY, USA) according to the manufacturer's instructions.

Chromatin immunoprecipitation. We performed ChIP using the EZ ChIP chromatin immunoprecipitation kit for cell line samples (Millipore). Briefly, we sonicated the crosslinked chromatin DNA into 200- to 500-bp fragments. The chromatin was then immunoprecipitated using an anti-methyl-histone H3 antibody and EZH2 (1:5000). Normal mouse IgG was used as the negative control. The primer sequences are listed in Supplementary Table 1. The antibodies for the ChIP assays of EZH2 and H3K27 were obtained from Millipore. Quantification of the immunoprecipitated DNA was performed using GPCR with SYBR Green Mix (TaKaRa). The ChIP data were calculated as a percentage relative to the input DNA using the equation 2: input $\mathrm{Ct}-$ Target $\mathrm{Ct} \times 0.1 \times 100$.

RNA immunoprecipitation. We performed RIP experiments using the Magna RIP RNA-binding protein immunoprecipitation kit (Millipore) according to the manufacturer's instructions. The antibodies for the RIP assays of EZH2 were obtained from Abcam. The co-precipitated RNAs were detected by reversetranscription PCR. The total RNAs were the input controls.
Tail vein injections into athymic mice. We purchased athymic male mice (4 weeks old) from the Animal Center of the Chinese Academy of Science (Shanghai, China) and housed them in laminar flow cabinets under specific pathogen-free conditions. Sh-HOTAIR stably transfected BGC-823 cells were selected by using G418 (400 $\mu \mathrm{g} / \mathrm{ml})$. BGC-823 cells transfected with HOTAIR1\# and pENTR vector (EV) were harvested from six-well plates, washed with phosphatebuffered saline (PBS), and resuspended at $2 \times 10^{7}$ cells per $\mathrm{ml}$. The resuspended cells $(0.1 \mathrm{ml})$ were injected into the tail veins of seven mice, which were killed 7 weeks after injection. The lungs were removed and photographed, and visible tumors on the lung surface were counted. This study was performed in strict accordance with the Guide for the Care and Use of Laboratory Animals of the National Institutes of Health. The Committee on the Ethics of Animal Experiments of Nanjing Medical University approved our protocol (Permit Number: 200933). All surgery was performed under sodium pentobarbital anesthesia, and all efforts were made to minimize suffering.

Fluorescence immunohistochemistry. Cells were fixed in $4 \%$ paraformaldehyde following a standard protocol. A rabbit anti-Snail polyclonal antibody (1:50; Abcam) was used as a primary antibody, with TRITC-labeled anti-rabbit lgG (1: 500; Sigma-Aldrich) used as a secondary antibody. Sections were mounted onto slides using Gel Mount aqueous mounting medium (G0918, Sigma-Aldrich) and examined with an Olympus BX51 microscope (Olympus Optical). Cells were fixed with $4 \%$ formaldehyde and examined by fluorescence microscopy to detect fluorescence intensity of the cells counted. Fluorescence intensity of the cells was measured using ImageJ (National Institutes of Health, Bethesda, MD, USA).

Bioinformatics methods. GSEA software was downloaded from Broad Institute (http://www.broadinstitute.org/gsea/index.jsp). Gene profiling data downstream HOTAIR were obtained from Gene Expression Omnibus (GEO) site (http:// www.ncbi.nIm.nih.gov/geo/query/acc.cgi?acc = GSE47638). Significantly enriched gene sets were identified, which produced a nominal $P$-value 0.05 . UCSC Genome Browser (http://genome.ucsc.edu/cgi-bin/hgGateway) was used to analyze promoter regions.

Statistical analysis. The SPSS 17.0 statistical analysis software (Armonk, NY, USA) was used for the statistical analysis of the experimental data. The significance of differences between groups was estimated by Student's $t$-test and the $\chi^{2}$ test. The levels of HOTAIR in the gastric cancer patients were compared using the MannWhitney $U$ test. The levels of HOTAIR, CDH1 and miR34a in the gastric cancer patients were assessed by the Spearman's correlation analysis. The disease-free survival probability was analyzed using Kaplan-Meier methods and evaluated using the log-rank test. A $P$-value $<0.05$ were considered significant.

\section{Conflict of Interest}

The authors declare no conflict of interest.

Acknowledgements. This work was supported by grants from the National Natural Science Foundation of China (No. 81472198), the Key Clinical Medicine Technology Foundation of Jiangsu Province (No. BL2014096) and the Medical Key Talented Person Foundation of the Jiangsu Provincial Developing Health Project (No. RC2011080) to Z-xW.

1. Cancer Statistics 2013

2. Orimo T, Nakano S, Inagaki M, Akabane $\mathrm{H}$, Yanagida $\mathrm{N}$, Shibaki $\mathrm{T}$ et al. [A case of AFPproducing gastric cancer patient with liver relapse occurred three months after endoscopic mucosal resection (EMR) and gastrectomy]. Gan to Kagaku Ryoho 2011; 38: 2328-2330.

3. Thiery JP, Acloque H, Huang RY, Nieto MA. Epithelial-mesenchymal transitions in development and disease. Cell 2009; 139: 871-890.

4. Gao D, Vahdat LT, Wong S, Chang JC, Mittal V. Microenvironmental regulation of epithelialmesenchymal transitions in cancer. Cancer Res 2012; 72: 4883-4889.

5. Acloque H, Adams MS, Fishwick K, Bronner-Fraser M, Nieto MA. Epithelial-mesenchymal transitions: the importance of changing cell state in development and disease. J Clin Invest 2009; 119: 1438-1449.

6. Perl AK, Wilgenbus P, Dahl U, Semb H, Christofori G. A causal role for E-cadherin in the transition from adenoma to carcinoma. Nature 1998; 392: 190-193.

7. Davalos V, Moutinho C, Villanueva A, Boque R, Silva $P$, Carneiro F et al. Dynamic epigenetic regulation of the microRNA-200 family mediates epithelial and mesenchymal transitions in human tumorigenesis. Oncogene 2012; 31: 2062-2074. 
8. Kiesslich T, Pichler M, Neureiter D. Epigenetic control of epithelial-mesenchymal-transition in human cancer. Mol Clin Oncol 2013; 1: 3-11.

9. Tam WL, Weinberg RA. The epigenetics of epithelial-mesenchymal plasticity in cancer. Nat Med 2013; 19: 1438-1449.

10. Siemens H, Jackstadt R, Hunten S, Kaller M, Menssen A, Gotz U et al. miR-34 and SNAIL form a double-negative feedback loop to regulate epithelial-mesenchymal transitions. Cell Cycle 2011; 10: 4256-4271.

11. Siemens H, Jackstadt R, Hünten S, Kaller M, Menssen A, Götz U et al. miR-34 and SNAIL form a double-negative feedback loop to regulate epithelial-mesenchymal transitions. Cell Cycle 2011; 10: 4256-4271.

12. Rokavec M, Öner MG, Li H, Jackstadt R, Jiang L, Lodygin D et al. IL-6R/STAT3/miR-34a feedback loop promotes EMT-mediated colorectal cancer invasion and metastasis. J Clin Invest 2014; 124: 1853-1867.

13. Sun M, Liu XH, Wang KM, Nie FQ, Kong R, Yang JS et al. Downregulation of BRAF activated non-coding RNA is associated with poor prognosis for non-small cell lung cancer and promotes metastasis by affecting epithelial-mesenchymal transition. Mol Cancer 2014; 13 68 .

14. Mattick JS. The genetic signatures of noncoding RNAs. PLoS Genet 2009; 5: e1000459.

15. Tsai MC, Spitale RC, Chang HY. Long intergenic noncoding RNAs: new links in cancer progression. Cancer Res 2011; 71: 3-7.

16. Spizzo R, Almeida MI, Colombatti A, Calin GA. Long non-coding RNAs and cancer: a new frontier of translational research? Oncogene 2012; 31: 4577-4587.

17. Rinn JL, Kertesz M, Wang JK, Squazzo SL, Xu X, Brugmann SA et al. Functional demarcation of active and silent chromatin domains in human HOX loci by noncoding RNAs. Cell 2007; 129: 1311-1323.

18. Kim K, Jutooru I, Chadalapaka G, Johnson G, Frank J, Burghardt R. HOTAIR is a negative prognostic factor and exhibits pro-oncogenic activity in pancreatic cancer. Oncogene 2012 32: $1616-1625$.

19. Kogo R, Shimamura T, Mimori K, Kawahara K, Imoto S, Sudo T et al. Long noncoding RNA HOTAIR regulates polycomb-dependent chromatin modification and is associated with poor prognosis in colorectal cancers. Cancer Res 2011; 71: 6320-6326.

20. Gupta RA, Shah N, Wang KC, Kim J, Horlings HM, Wong DJ et al. Long non-coding RNA HOTAIR reprograms chromatin state to promote cancer metastasis. Nature 2010; 464 1071-1076.

21. Niinuma T, Suzuki H, Nojima M, Nosho K, Yamamoto H, Takamaru H et al. Upregulation of miR-196a and HOTAIR drive malignant character in gastrointestinal stromal tumors. Cancer Res 2012; 72: 1126-1136.

22. Liu XH, Sun M, Nie FQ, Ge YB, Zhang EB, Yin DD et al. Lnc RNA HOTAIR functions as a competing endogenous RNA to regulate HER2 expression by sponging miR-331-3p in gastric cancer. Mol Cancer 2014; 13: 92

23. Chase A, Cross NC. Aberrations of EZH2 in cancer. Clin Cancer Res 2011; 17: 2613-2618.

24. Gieni RS, Hendzel MJ. Polycomb group protein gene silencing, non-coding RNA, stem cells, and cancer. Biochem Cell Biol 2009; 87: 711-746.

25. Goyal L, Muzumdar MD, Zhu AX. Targeting the HGF/c-MET pathway in hepatocellular carcinoma. Clin Cancer Res 2013; 19: 2310-2318.

26. Gherardi E, Birchmeier W, Birchmeier C, Woude GV. Targeting MET in cancer: rationale and progress. Nat Rev Cancer 2012; 12: 89-103.

27. Grotegut S, von Schweinitz D, Christofori G, Lehembre F. Hepatocyte growth factor induces cell scattering through MAPK/Egr-1-mediated upregulation of Snail. EMBO J 2006; 25 3534-3545.

28. Wang Y, Shi J, Chai K, Ying X, Zhou BP. The role of Snail in EMT and tumorigenesis. Curr Cancer Drug Targets 2013; 13: 963-972.

29. Henson DE, Dittus C, Younes M, Nguyen H, Albores-Saavedra J. Differential trends in the intestinal and diffuse types of gastric carcinoma in the United States, 1973-2000: increase in the signet ring cell type. Arch Pathol Lab Med 2004; 128: 765-770.

30. Cao WJ, Wu HL, He BS, Zhang YS, Zhang ZY. Analysis of long non-coding RNA expression profiles in gastric cancer. World J Gastroenterol 2013; 19: 3658-3664.

31. Li H, Yu B, Li J, Su L, Yan M, Zhu Z et al. Overexpression of IncRNA H19 enhances carcinogenesis and metastasis of gastric cancer. Oncotarget 2014; 5: 2318-2329.

32. Christiansen JJ, Rajasekaran AK. Reassessing epithelial to mesenchymal transition as a prerequisite for carcinoma invasion and metastasis. Cancer Res 2006; 66: 8319-8326.
33. Fuchs BC, Fujii T, Dorfman JD, Goodwin JM, Zhu AX, Lanuti M et al. Epithelial-tomesenchymal transition and integrin-linked kinase mediate sensitivity to epidermal growth factor receptor inhibition in human hepatoma cells. Cancer Res 2008; 68: 2391-2399.

34. Cheng GZ, Chan J, Wang Q, Zhang W, Sun CD, Wang LH. Twist transcriptionally up-regulates AKT2 in breast cancer cells leading to increased migration, invasion, and resistance to paclitaxel. Cancer Res 2007; 67: 1979-1987.

35. Savagner P. The epithelial-mesenchymal transition (EMT) phenomenon. Ann Oncol 2010; 21(Suppl 7): i89-i92.

36. Yang J, Weinberg RA. Epithelial-mesenchymal transition: at the crossroads of development and tumor metastasis. Dev Cell 2008; 14: 818-829.

37. Peinado $\mathrm{H}$, Olmeda D, Cano A. Snail, Zeb and bHLH factors in tumour progression: an alliance against the epithelial phenotype? Nat Rev Cancer 2007; 7: 415-428.

38. Zhang JP, Zeng C, Xu L, Gong J, Fang JH, Zhuang SM. MicroRNA-148a suppresses the epithelial-mesenchymal transition and metastasis of hepatoma cells by targeting Met/Snail signaling. Oncogene 2014; 33: 4069-4076.

39. Burk U, Schubert J, Wellner U, Schmalhofer O, Vincan E, Spaderna S et al. A reciprocal repression between ZEB1 and members of the miR-200 family promotes EMT and invasion in cancer cells. EMBO Rep 2008; 9: 582-589.

40. Ying L, Chen Q, Wang Y, Zhou Z, Huang Y, Qiu F. Upregulated MALAT-1 contributes to bladder cancer cell migration by inducing epithelial-to-mesenchymal transition. Mol Biosyst 2012; 8: 2289-2294.

41. Jeggari A, Marks DS, Larsson E. miRcode: a map of putative microRNA target sites in the long non-coding transcriptome. Bioinformatics 2012; 28: 2062-2063.

42. Braconi C, Kogure T, Valeri N, Huang N, Nuovo G, Costinean S et al. microRNA-29 can regulate expression of the long non-coding RNA gene MEG3 in hepatocellular cancer. Oncogene 2011; 30: 4750-4756.

43. Khalil AM, Guttman M, Huarte M, Garber M, Raj A, Rivea MD et al. Many human large intergenic noncoding RNAs associate with chromatin-modifying complexes and affect gene expression. Proc Natl Acad Sci USA 2009; 106: 11667-11672.

44. Au SL, Wong CC, Lee JM, Fan DN, Tsang FH, Ng IO et al. Enhancer of zeste homolog 2 epigenetically silences multiple tumor suppressor microRNAs to promote liver cancer metastasis. Hepatology 2012; 56: 622-631.

45. Bao B, Ali S, Banerjee S, Wang Z, Logna F, Azmi AS et al. Curcumin analogue CDF inhibits pancreatic tumor growth by switching on suppressor microRNAs and attenuating EZH2 expression. Cancer Res 2012; 72: 335-345.

46. Matsukawa Y, Semba S, Kato H, Ito A, Yanagihara K, Yokozaki H. Expression of the enhancer of zeste homolog 2 is correlated with poor prognosis in human gastric cancer. Cancer Sci 2006; 97: 484-491.

47. Welch $\mathrm{C}$, Chen Y, Stallings RL. MicroRNA-34a functions as a potential tumor suppressor by inducing apoptosis in neuroblastoma cells. Oncogene 2007; 26: 5017-5022.

48. Li Y, Guessous F, Zhang Y, Dipierro C, Kefas B, Johnson E et al. MicroRNA-34a inhibits glioblastoma growth by targeting multiple oncogenes. Cancer Res 2009; 69: 7569-7576.

49. Zauli G, Voltan R, di lasio MG, Bosco R, Melloni E, Sana ME et al. miR-34a induces the downregulation of both E2F1 and B-Myb oncogenes in leukemic cells. Clin Cancer Res 2011; 17: 2712-2724

50. Yu S, Yu Y, Zhao N, Cui J, Li W, Liu T. c-Met as a prognostic marker in gastric cancer: a systematic review and meta-analysis. PLoS One 2013; 8: e79137.

(i) Cell Death and Disease is an open-access journal published by Nature Publishing Group. This work is licensed under a Creative Commons Attribution 4.0 International License. The images or other third party material in this article are included in the article's Creative Commons license, unless indicated otherwise in the credit line; if the material is not included under the Creative Commons license, users will need to obtain permission from the license holder to reproduce the material. To view a copy of this license, visit http://creativecommons.org/licenses/by/4.0/ 